\title{
A VISUALIZATION MODEL OF FLOWER BASED ON DEFORMATION
}

\author{
Ling Lu ${ }^{1, *}$, Lei Wang ${ }^{1}$, Xuedong Yang ${ }^{2}$ \\ ${ }^{1}$ School of Information Engineering, East China Institute of Technology, JiangXi Province, P. \\ R. China 344000 \\ ${ }^{2}$ Department of Computer Science, University of Regina, Regina, Saskatchewan Canada \\ S4S $0 A 2$ \\ * Corresponding author, Address: School of Information Engineering, East China Institute of \\ Technology, FuZhou344000, JiangXi Province, P. R. China, Tel: +86-794-8258390, Fax: \\ +86-794-8258390, Email: luling@ecit.edu.cn
}

Abstract: We present a simple and effective modeling method for flowers. It starts with an initial geometric shape, such as ellipsoid, cylinder, or plane surface et al., and then simulates flower components (such as pedicel, receptacle, pistils, stamens, petals and sepals) by addition deformation to the basic geometric shape. The detailed geometry of flower component is defined by basic equation for the basic shape along with a deformation function. A variety of flower can be produced by varying the deformation parameters. A number of examples are given in the paper to demonstrate the effectiveness of the proposed model.

Keywords: plant flower, basic geometric shape, deformation

\section{INTRODUCTION}

Flowers pose an interesting and important challenge for three-dimensional computer graphics modeling. They have a great number of components, such as stems, torus, pistils, stamens, petals. They also have intricate structure and unique free-form. The L-System was first formulated by Lindenmayer (1968) and was introduced to the computer graphics community by Prusinkiewicz and Lindenmayer(1990). The L-System defines plant structures using a set of rewriting rules (Prusinkiewicz et al.,1990). Prusinkiewicz et al.

Please use the following format when citing this chapter:

Lu, L., Wang, L. and Yang, X., 2009, in IFIP International Federation for Information Processing, Volume 294, Computer and Computing Technologies in Agriculture II, Volume 2, eds. D. Li, Z. Chunjiang, (Boston: Springer), pp. 1487-1495. 
(2001) also used the positional information to control parameters along a plant's axis. Prusinkiewicz et al. (1993) introduced dL-System by combining differential equations with the L-System. The dL-System provides a model that simulates plant growth in continuous time. These studies, however, mainly focused on branching structure or skeletal shape, and did not deal with the growth of surface tissues of plants, such as petals or leaves. Recently, some studies published methods dealing with plant surfaces. Runions et al. (2005) presented an algorithm for generating leaf venation patterns based on the canalization hypothesis. The algorithms can simulate many types of venation patterns. The initial leaf shape is specified interactively by the user, as a parametric curve that defines the leaf contour. Wang et al. (2004) considered leaf tissues to be viscous and simulated growth by expansion of incompressible fluids. Lintermann et al (1999) presented a modeling method that allows easy generation of many types of objects that have branching structures, including flowers, bushes, trees, and even some non-botanical objects. Flower's natural leaf was scanned and applied as a texture to the leaf component's surface. Their three-dimensional appearance was not satisfactory because of the use of two-dimensional texture. Recently, Wang et al (2005) presented a framework for real-time rendering of plant leaves with global illumination effects. Their leaf model can be captured from real leaves, which makes it easy to create highly realistic leaf appearance models. But their leaf model did not consider small features such as hairs on leaves. Ijiri et al. (2005) presented a system for modeling flowers in three dimensions quickly and easily while preserving correct botanical structures. This system is an application-customized sketch-based interface. The user needs to input the outline of petal and draws modifying strokes. The petal shapes that can be generated by this system are limited to elliptical outlines. Qin Peiyu et al. (2006) proposed a flower model using the L- system and Bezier surfaces. One of the drawbacks of using Bezier surfaces is the lack of ability to model fine concave or convex texture on petals and toothed borderlines.

We use our deformation technology ( $\mathrm{Lu}$ Ling et al.2007) to form flower's components, such as stems, torus, pistils, stamens, and petals. A number of examples are given in the paper to demonstrate the effectiveness of the proposed model.

\section{GEOMETRY MODELS FOR FLOWER COMPONENTS}

Flower is composed of pedicel, receptacle, pistils, stamens, petals and sepals. Pistil consists of stigma, style and ovary. Stamen consists of anther and filament. They is shown in Fig.1(Hu Baozhonf et al.2002 ) 


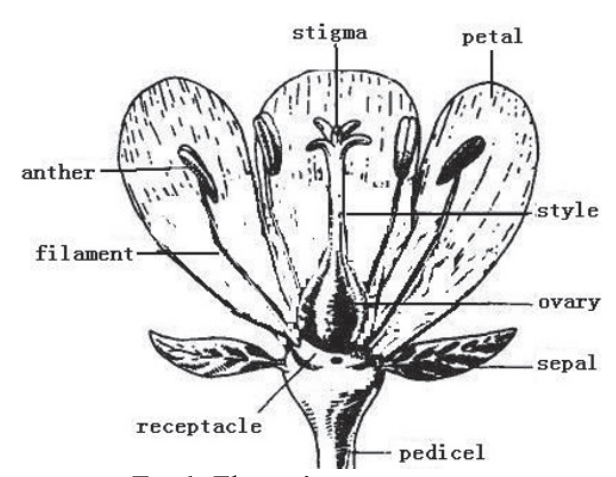

Fig. 1: Flower's component.

\section{1 $\quad$ Pedicel modeling}

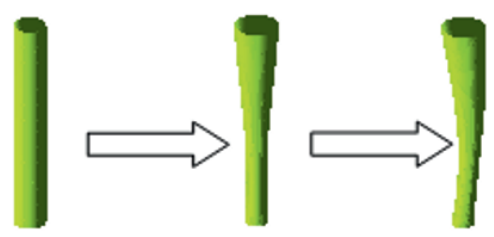

Fig.2: The simulator process of Pedicel

The pedicel is a small spray with flower and its structure is similar to stem. Its length and thickness is different from a plant. Since its shape looks likes cylinder. We may deform a cylinder to simulate pedice1 (Fig.2). The cylinder is represented as the follows:

$$
\begin{aligned}
& x(u, v)=r \cos (u) \\
& y(u, v)=h v \\
& z(u, v)=r \sin (u)
\end{aligned} \quad(0 \leqslant \mathrm{u} \leqslant 2 \pi, 0 \leqslant \mathrm{v} \leqslant 1)
$$

where $r$ is radius of the cylinder, $h$ is height of the cylinder. We add deformation function to make cylinder uneven and bend:

$$
\begin{aligned}
& x(u, v)=r \cos (u)+n_{x} a_{1} \sin (\pi v-1)+a_{2} \sin (\pi v) \\
& y(u, v)=h v+n_{y} a_{1} \sin (\pi v-1) \quad(0 \leqslant \mathrm{u} \leqslant 2 \pi, 0 \leqslant \mathrm{v} \leqslant 1) \\
& z(u, v)=r \sin (u)+n_{z} a_{1} \sin (\pi v-1)+a_{2} \sin (\pi v)
\end{aligned}
$$

where $a_{1}$ is the extent of uneven deformation, $a_{2}$ is the extent of bend deformation, and $\left(n_{x}, n_{y}, n_{z}\right)$ is unit surface normal at the point $(x, y, z)$. In Fig.2, both $a_{1}$ and $a_{2}$ are equal 3 . 


\section{$2.2 \quad$ Receptacle modeling}

The receptacle is at the top of pedicel. The flower components grow on a receptacle with special layout. There are many kinds of shape for receptacle, such as cylinder, cone, bowl etc. (Fig.3)

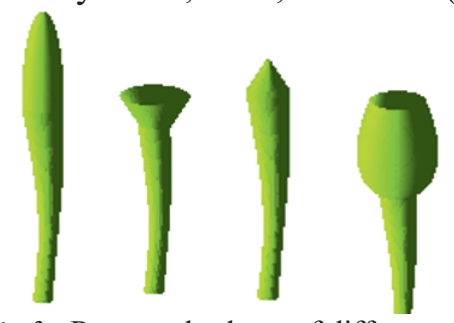

Fig.3: Receptacle shape of different

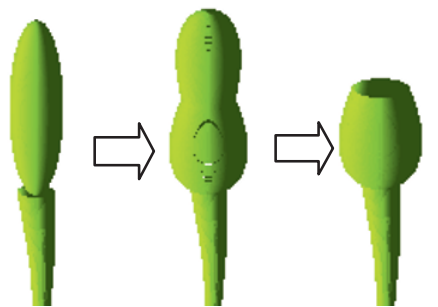

Fig4: The simulator process of the bowl shape

For example, the bowl shape comes from ellipsoid deformation (Fig.4). First, the parametric ellipsoid equation is as the follows (Fig.4(a))

$$
\begin{aligned}
& x(u, v)=r \cos (u) \cos (v) \\
& y(u, v)=3 r \sin (u) \\
& z(u, v)=r \cos (u) \sin (v)
\end{aligned} \quad(-\pi / 2 \leqslant \mathrm{u} \leqslant \pi / 2,0 \leqslant \mathrm{v} \leqslant 2 \pi)
$$

Second, we modify the surface-position vector by adding a deformation function (Lu ling et al.2007).We have (Fig.4(b))

$$
\begin{aligned}
& x(u, v)=r \cos (u) \cos (v)+n_{x} r / 2|\sin (2 u)| \\
& y(u, v)=3 r \sin (u)+n_{y} r / 2|\sin (2 u)| \quad(0 \leqslant \mathrm{u} \leqslant \pi / 2,0 \leqslant \mathrm{v} \leqslant 2 \pi) \\
& z(u, v)=r \cos (u) \sin (v)+n_{z} r / 2|\sin (2 u)| \\
& x(u, v)=r \cos (u) \cos (v)+n_{x} r / 1.4|\sin (2 u)| \\
& y(u, v)=3 r \sin (u)+n_{y} r / 1.4|\sin (2 u)| \quad(-\pi / 2 \leqslant \mathrm{u} \leqslant 0,0 \leqslant \mathrm{v} \leqslant 2 \pi) \\
& z(u, v)=r \cos (u) \sin (v)+n_{z} r / 1.4|\sin (2 u)|
\end{aligned}
$$

Finally, when $0 \leqslant u \leqslant \pi / 2$, we add a function $-r \cos (\pi / 2-u)$ to $z(u, v)(\operatorname{Eq}(4))$. The upper part of deform ellipsoid is changed to inside of bowl (Fig,4(c)).

\subsection{Pistil and stamen modeling}

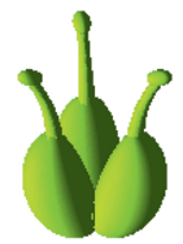

(a) apocarpous gynoecium

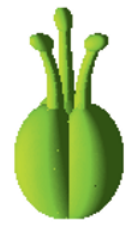

(b)compound pistil

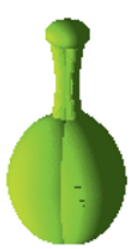

(c)compound pistil

Fig.5: Different kind of pistil 
Pistil is formed by carpel intervolve. The type of pistil is determined by the number of carpel, and extent of separation. Simple pistil consists of a carpel. Many carpels construct a compound pistil. In some plant, a flower has a lot of carpel such that they are apart and form respective pistil. These pistils are named apocarpous gynoecium (Fig.5(a)). Every pistil is divided into three parts: stigma, style and ovary. We may use ellipsoid to simulate stigma, deformation cylinder to simulate style, and deformation ellipsoid to simulate ovary. In Fig.5(a), an ovary model follows

$$
\begin{aligned}
& x(u, v)=r \cos (u) \cos (v) \\
& y(u, v)=2 r \sin (u) \\
& (-\pi / 2 \leqslant \mathrm{u} \leqslant \pi / 2,0 \leqslant \mathrm{v} \leqslant 2 \pi) \\
& z(u, v)=r \cos (u) \sin (v)-r / 2 \sin (\pi / 2+u)
\end{aligned}
$$

A style equation is

$$
\begin{aligned}
& x(u, v)=r \cos (u)+n_{x} \sin (\pi v-1) \\
& y(u, v)=10 r v+n_{y} \sin (\pi v-1) \\
& z(u, v)=r \sin (u)+3 \sin (\pi v)+n_{z} \sin (\pi v-1)
\end{aligned} \quad(0 \leqslant \mathrm{u} \leqslant 2 \pi, 0 \leqslant \mathrm{v} \leqslant 1)
$$

A stigma may be expressed by ellipsoid. So we may combine a stigma, a style and an ovary to form a pistil. For apocarpous gynoecium, we use many several independent pistils with different rotation angles. The compound pistil is constructed by several pistils that are closely staggered (Fig 5(b)).

Every stamen consists of an anther and a filament. The anther, expanded to become a saccate, is at top of filament. We use a deformed ellipsoid to simulate anther. Since the shape of filament is long and thin, it may be made by deformation cylinder. Depending on the separation between the stamens and the different length of stamens, a stamen is divided into several types: monadelphous stamen, diadelphous stamens, polydelphous stamens, tetradynamous stamen, didynamous stamen and synantherous stamen. The methods for their simulation are similar to pistil.

\subsection{Petal and sepal modeling}

The shape of petal and sepal are similar. Therefore, we use petal as an example to describe the method. Because petal is a curve surface, we select the initial shape of a petal is a rectangle surface. We add deformation function ( $\triangle x$ and $\triangle y$ ) to form free curve outline and modify the surfaceposition vector by adding a deformation function $(\triangle g)$. We have (Lu Ling et al., 2008)

$$
\begin{aligned}
& x(u, v)=a_{x} u+\Delta x+n_{x} \Delta g \\
& y(u, v)=b_{y} v+\Delta y+n_{y} \Delta g \\
& z(u, v)=c_{z}+n_{z} \Delta g
\end{aligned}
$$


where $a_{x}$ is the length with respect to the x direction, $b_{y}$ is the length along the y direction, and $c_{\mathrm{z}}$ is the section distance.

For example, the deformation function of peony petal is as follows

$$
\begin{aligned}
\Delta x= & 0.8 a_{x} u \sin (\pi v / 2-c) \\
\Delta y= & (v-0.5)\left(1.5 a_{x} \sin (\pi(u+0.5))+\mid 3 v \sin \left(\pi a_{x}(u+0.5) / 6\right)\right. \\
\Delta g= & 0.3(1-2|v-0.5|) \sin (20(u+0.5) \pi)(1-2|u|)+3(1-v)^{2} e^{-800 u^{2}}+ \\
& d y \sin (\pi y / 40-1)+d x \sin (\pi x / 2-0.5)+2 v^{3} \sin (\pi x / 8)
\end{aligned}
$$

The process from rectangle surface to a peony petal is showed in Fig.6.

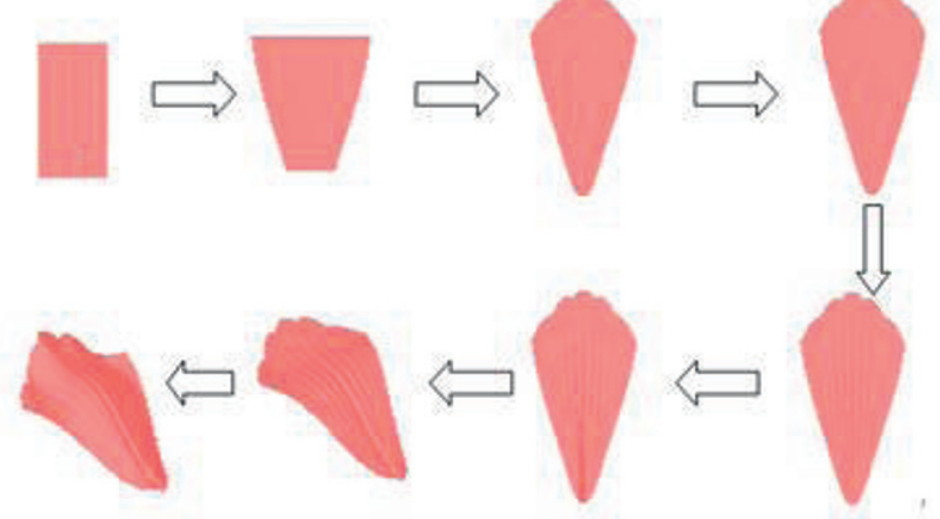

Fig. 6: The process from rectangle surface to peony petal

\section{FLOWER MODELING}

\subsection{Flower color}

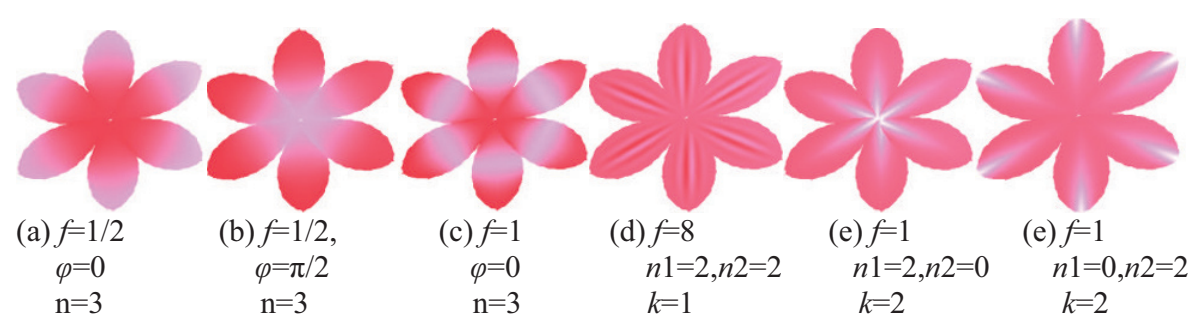

Fig.7: The pattern model of multicolored

The color of flower is divided into two types (Zhao et al., 2005): one is single color, another is multicolored. Generally, a pedicel and a receptacle are single color. The composition of pistil and stamen, such as stigma, style and ovary, are also single color. But a petal is multicolored and its color 
model is more complex. In common, the pattern model of multicolored include flower centre, flower side, flower ring and flower rib etc (Zhou et al., 2007) (Fig 7). Maybe a kind of flower has many pattern models. For simplification, we use basic function to simulate a petal multicolored. For example, let the intensity vary from $I_{1}$ to $I_{2}\left(I_{1}<I_{2}\right)$ with respect to $\mathrm{v}$ as the function of

$$
I=I_{1}+I_{2} \sin ^{n}(v \pi f+\varphi)
$$

where $n$ controls the change rate from $I_{1}$ to $I_{2}$. Fig $7(\mathrm{a}-\mathrm{c})$ shows the result of different value in Eq.(9). For flower rib, we may create a function as follows

$$
I=I_{1}+I_{2} \sin (f(u+0.5) \pi)(1-v)^{n 1} v^{n 2}(1-2|u|)^{k}
$$

where $f$ controls the number of rib, $n 1, n 2$ and $k$ control the border rib of a petal. Fig 7(d-f) shows the result of different value in Eq.(10).

\subsection{Synthesis flower components}

After flower components have been modeled, we may build an entire flower model. Based on the characteristics of a flower, we may combine flower component (such as pedicel, receptacle, pistils, stamens, petals and sepals) accordingly. The number of individual component is different from one flower to another. For example, a lily has a pistil, six stamens and six petals. A peony has many stamens and many petals. Every petal is also not same. The inside petals are bigger and more bender than outside petals. The size and bent of a petal can be controlled easily by our deformation definition. Fig.8 show that we can use different parameter in Eq.(7) and Eq.(8) to construct different petal shape.

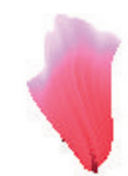

$$
\begin{aligned}
\text { (a) } a_{x}=15 \\
b_{y}=40 \\
c=0.4 \\
d x=-7 \\
d y=5
\end{aligned}
$$

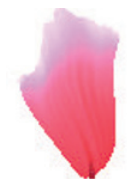

(b) $a_{x}=18$

$b_{y}=41$

$c=0.2$

$d x=-6$

$d y=4$

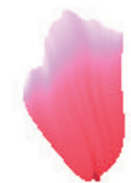

(c) $a_{x}=21$

$$
b_{y}=42
$$

$c=0$

$d x=-5$

$d y=3$

Fig. 8

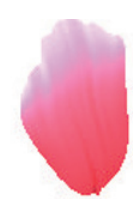

(d) $a_{x}=24$

$b_{y}=43$

$c=-0.2$

$d x=-4$

$d y=2$

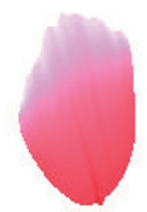

(e) $a_{x}=27$

$b_{y}=44$

$c=-0.4$

$d x=-3$

$d y=1$

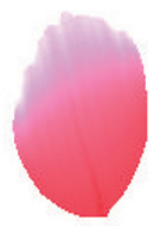

(f) $a_{x}=30$

$b_{y}=45$

$c=-0.6$

$d x=-2$

$d y=0$

We place geometric objects on the receptacle model. For peony petal, we may use a recursion iteration or L-system to simulate many stamens and petals (Fig.8).The position of stamens and petals are determined by random number. 

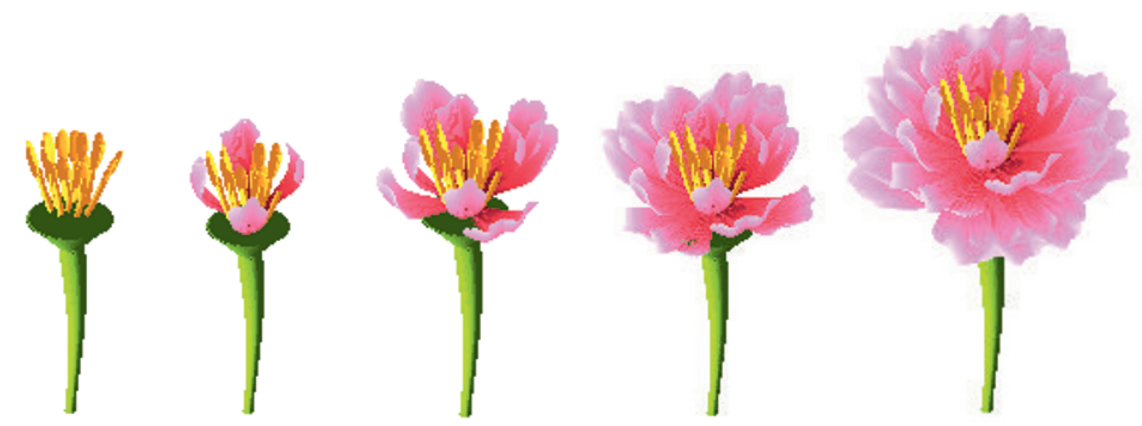

Fig.9: Peony model

\section{CONCLUSION}

In this paper, we presented a modeling method for flowers using deformation. This method start with basic geometric shape, such as ellipsoid, cylinder and rectangle surface, and add deformation function, making a basic geometric shape to approach a desired flower component shape. The geometric model of a flower can be controlled through a small set of parameters. Because our models are simple mathematical expressions controlled by a few parameters, these parameters may be changed in a continuous manner, such that both the shape and color of the flower can vary continuously. A growth model based the current deformation technique will be our future research in the next step.

\section{REFERENCES}

Hu Paozhong, Hu Guoxuan. Botanical. China Agricultural Publishing 2002,3

Ijiri T Owada S Okabe $\mathrm{M}$ et al. Floral diagrams and inflorescences : interactive flower modeling using botanical structural constraints[C] PPComputer Graphics Proceedings , Annual Conference Series, ACM SIGGRAPH , Los Angles , 2005 :720 -726.

Lindenmayer A.: Mathematical models for cellular interactions in development, I \& II. Journal of Theoretical Biology 18, 3 (1968),280-315.

Lintermann B Deussen O. Interactive modeling of plants [J]. IEEE Computer Graphics \& Applications , 1999 (1):56-65.

$\mathrm{Lu}$ Ling Wang Lei. Visualization Model of Plant Petal Based on Plane Deformation. Transactions of the Chinese Society for Agricultural Machinery. 2008. (9)

Lu Ling, Xu Hongzhen, Song Wenlin, Liu Gelin. Research on Visualization of Plant Fruits Based on Deformation. New Zealand Journal of Agricultural Research.2007.11

Prusinkiewicz P., Hammel, M., Mjolsness E.: Animation of plant development. In Proc. IGGRAPH '93, 351-360.

Prusinkiewicz P., Lindenmayer A.: The Algorithmic Beauty of Plants. Springer-Verlag, New York, 1990. With J. S. Hanan, F. D. Fracchia,D. R. Fowler, M. J. M. de Boer, and L. Mercer. 
Prusinkiewicz P., Mündermann L., Karwowski R., Lane B.: The use of positional information in the modeling of plants. In Proc. ACM SIGGRAPH '01 (2001), 289-300.

Qin Peiyu, Chen Chuanbo, Lv Zehua. Simulation Model of Flower Using the Interaction of Lsystems with Bezier Surfaces[J].Computer Engineering and application. 2006.16,pp:6-8.

Runions A., Fuhrer M., Lane B., Federl P., Rolland-Lagan A.-G.,and Prusinkiewicz P.: Modeling and visualization of leaf venation patterns. ACM Trans. Graph., 24, 3 (2005), $702-711$.

Wang I., Wan J., Baranoski, G.: Physically-based simulation of plant leaf growth. Computer Animation and Virtual Worlds, 15, 3-4 (2004), 237-244.

Wang L Wang W Dorsey J et al. Real-time rendering of plant leaves [C] PPComputer Graphics Proceedings, Annual Conference Series, ACM SIGGRAPH, Los Angles , 2005:712-719.

Zhao Changling, Guo Weiming, Chen Junyu. Formation and regulation of flower color in higher plants [J].Chinese Bulletin of Botany , 2005, 22 (1) : 70 - 81

Zhou Ning,Dong Weiming,Wang Jiaxin, Simulation of Flower Color Pattern[J], Journal of Computer-aided Design \& Computer Graphics. 2007, 19(6):708-712 